\title{
Advance Technique for Feature Extraction and Image Compression
}

\author{
Arvind Kourav \\ Electronics \& Communication Engineering \\ Research Scholar D.K.N.M.U. Niwai Rajasthan India
}

\author{
Prashant Singh, PhD. \\ Computer Science \& Engineering \\ ISC Software Pvt. Ltd., Bhopal India
}

\begin{abstract}
For image processing, it is very necessary that the selection of transform. In this paper, a comparative analysis of curve let transform with other transform for image processing. In this we proposed the applications of curve let transform in the field of image Compression, phase recognition and feature extraction. For higher compression with quality reconstruction .The Wavelets gave a different aspect to the compression. Curvelet Transform gives better results in terms of PSNR. Face recognition is very important for many applications such as: video surveillance, criminal investigations and forensic applications, secure electronic banking, mobile phones, credit cards, secure access to buildings. The curve let transform is a multi scale directional transform, which allows an almost optimal non adaptive sparse representation of objects with edges. Curve let have also proven useful in diverse fields beyond the traditional image processing application, Curvelet transform improve recognition accuracy with featature extraction extraction algorithms PCA, LDA,ICA and NMF.
\end{abstract}

\section{Keywords}

Image processing, Image Compression, Feature Extraction, Curvelet transform, Wavelet Transform.

\section{INTRODUCTION}

In the field of Image processing different types of method can be use for the analysis of image in different field such as De noising , Compression ,Face recognition ,Bio medical application etc. These analysis of image is based on the different types of transform is Fourier transform, Wavelet Transform, Curve let transform, Now a day Wavelet and Curvelet transform is used in all field of image processing.

\subsection{DIGITAL IMAGE PROCESSING SYSTEM}

A typical digital image processing system consists of image segmentation, feature extraction, pattern recognition, thresholding and error classification. Image processing aims at extracting the necessary information from the image. The image needs to be reduced to certain defining characteristics and the analysis of these characteristics gives the relevant information.

\subsection{CURVELET TRANSFORM}

To overcome the draw back of Wavelet Transform .Curve let Transform is developed, Curve let Transform is very effective modal that not only consider a multi scale Time Frequency local portion but also make use of the direction of features. It was developed by Candes and Donoho in 1999, there are two types of Curvelet transform is unequally spaced Fast Fourier Transform and wrapping based fast Curve let Transform, in curve let transform the width and length are related by the relation Width Length 2 that is known as parabolic or anisotropic scaling. Moreover, frame elements in curve let indexed by scale, location and orientation parameters in contrast to wavelets where elements have only scale and location. This transform can we used for both continuous and digital domain ,In this angle polar wedges or angle trapezoid window is used in frequency domain .Initially construction of Curve let transform is redesigned as fast discrete Curve let transform (FDCT) by Candes in 2006.This is second generation curve let transform is meant to be simpler to understand and use. DCT can be implemented by wrapping based fast discrete curve let at a given scale. At a given scale and orientation both the image and the curve let are transformed into Fourier domain. The product of curve let and the image are obtained in the fourier domain. Inverse FFT is applied to the above product to obtain a set of curve let coefficients. In order to perform IFT the trapezoidal wedge thus obtained from the frequency response of a curve let is wrapped into a rectangular support. The spectrum inside the wedge is tilted periodically. Thus the rectangular region collects the wedge's fragmented portions by periodic tilting.

The paper is structured as tracks: the Image Compression are discussed briefly in section II; section III describes feature extraction methods.The result analysis is discussed in section $\mathrm{IV}$ and section $\mathrm{V}$ forms the conclusion.

\section{IMAGE COMPRESSION}

Basic steps of the image compression is shown in fig. 1. All these steps are invertible, therefore lossless, except for the Quantize step. Quantizing is the process of reduction of the precision of the floating point values of the curvelet transform.

\begin{tabular}{|l|l|l|l|}
\hline $\begin{array}{l}\text { Input } \\
\text { Image }\end{array}$ & $\begin{array}{l}\text { Curvelet } \\
\text { Coefficient }\end{array}$ & $\begin{array}{l}\text { Quantiz } \\
\text { ation } \\
\text { process }\end{array}$ \\
\hline
\end{tabular}

Fig 1: Image Compression Block Diagram

Mostly, scalar quantization is used for quantization approach [16.18]. But, is significantly affects the bitrate every time a run of zeros is broken. All nonzero coefficients, independent of size, have the same negative impact in the run length encoder. Small coefficients enhance the image quality to a great extent and they still mess up the Huffman encoding as much as the big coefficients. Figure 2 shows the utilization of a proposed quantizer rather than a scalar quantization by which it is possible to block the smallest coefficients [19]. Longer runs of zeros are now attained, and this improves the performance of the Huffman encoder. The performance of the entire system is improved in terms of $\mathrm{psnr} / \mathrm{bpp}$ 


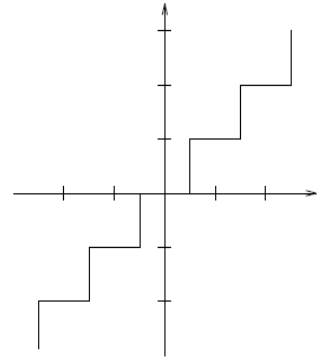

(a)

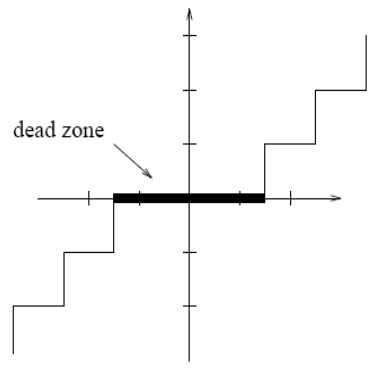

(b)
Fig 2: A Scalar Quantizer and a proposed Quantizer with a Dead Zone.

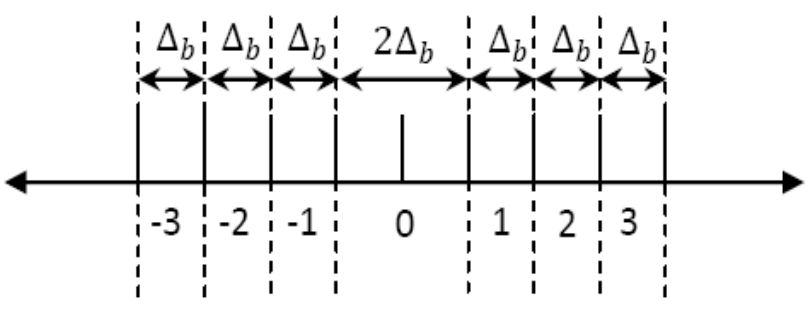

Fig 3: Dead-zone quantization about the origin.

\subsection{Proposed Method For Image \\ Compression}

The proposed image compression technique is very effective in overcome the limitations of the wavelet transform based coding techniques when applied to images with linear Curves. The steps involved in the proposed approach are:

i. Represent the pixels of image in the spatial co-ordinates.

ii. Apply Curvelet Transform on the image matrix and get the Curvelet coefficients of the image.

iii. Quantize the available coefficients using proposed Quantization Algorithm.

iv. Use Huffman coding on the bit stream.

The objective image compression method like Compression Ratio, Peak Signal to Noise Ratio (PSNR) and Root Mean Squared Error (RMSE).

Compression ratio: It is used to compute the minimization in image representation size produced by an image compression algorithm. The data compression ratio is equivalent to the physical compression ratio used to evaluate physical compression of substances and is defined as the ratio between the original image size and the compressed image size.

Root Mean Square Error (RMSE) metric is extensively used, having obvious physical interpretation and mathematically suitable. RMSE is computed by averaging the squared intensity difference of reconstructed image, the original image, . The RMSE is used to find the PSNR value of compress image.

\subsection{Proposed Algorithm For Quantizer}

The following steps are used for image compression using proposed quantizer of curvelet transform.

1. Initilize the input image

2. Find the features of the image

3. Apply compression algorithm using wavelet EZW
4. Apply curvelet transform in step 3 with proposed quantizer

5. Comparison of results for RMSE, PSNR And CR for Wavelet and Curvelet with proposed quantizer.

\section{Curvelet Based Feature Extraction for Faces}

A classic face recognition system consists of some key steps, namely, input face image collection, preprocessing,feature extraction, classification, and template storage or database.In the prior section, we have presented a theoritical overview of curvelet transform and explained why it can be expected to work better than the traditional wavelet transform. Facial images are generally 8 bit i.e. they have 256 gray levels. In such images two very close regions that have conflicting pixel values will give rise to edges; and these edges are typically curved for faces. As curvelets are good at approximating curved singularities, they are fit for extracting crucial edgebased features from facial images more efficiently than that compared to wavelet transform. We will now describe different face recognition algorithms that employ curvelet transform for feature extraction. Typically, a face recognition system is divided into two stages a training stage and a classification stage. In the training stage, a set of known faces (labeled data) are used to create a representative feature-set or template. In the classification stage, a unknown facial image is matched against the previously seen faces by comparing the features. Curvelet based feature extraction takes the raw or the preprocessed facial images as input. The images are then decomposed into curvelet subbands in different scales and orientations.Feature extraction algorithm is based on extracting spatial variations precisely from high-informative local zones of the face image instead of utilizing the entire image

\subsection{FACE RECOGNITION METHODS}

\section{A. Principle Component Analysis (PCA)}

1. Determine PCA subspace from training data. ith image vector containing $\mathrm{N}$ pixels is in the form

i] Store all images in the matrix

ii] Compute covariance matrix

iii] Compute eigenvalues and eigenvectors

corresponding eigenvalues in descending order. Keep only the eigenvectors associated with non- zero eigenvalues. This matrix of eigenvectors forms the eigen space, where each column of is the eigenvector. Visualized eigenvectors of the covariance matrix are called eigen faces [4] .PCA is a powerful tool for analysing data. The main advantage of PCA is to find the patterns in the data and reducing the number of dimensions without loss of information.

\section{B. Linear Discriminant Analysis (LDA)}

Linear Discriminant Analysis(LDA) is supervised learning technique because it needs class information for each image in the training process. LDA finds an efficient way to represent the face vector space by exploiting the class information [8]. It differentiates individual faces but recognize faces of the same individual .Basic steps of LDA algorithm LDA considers between a class correspondences of data. It means that training images create a class for each subject, ie, one class $=$ one subject (all his / her training images) [3]

1. Determine LDA subspace from training data. Calculate the within class scatter matrix and between class scatter matrix The following steps are performed by both methods. 
2. All training images are projected onto particular method's subspace

3. Each test image is also projected to the same subspace and compared by distance metrics between the image and training images.

\section{Independent Component Analysis}

ICA is a arithmetic method that represents a the analysis of random vector as a linear combination of non gaussian random variables ('independent components') that are as independent as possible.ICA is somewhat similar to PCA. Applications of ICA in data analysis and feature extraction ICA minimizes both second - order and higher -order dependencies in the input data and attempt to find the basis along which the data are statistically independent. There are two construction of ICA for face recognition [11] Architecture I - Statistically independent basis image. Architecture II factorial code representation.To obtain completely independent components, which constitute complete faces.

\section{ANALYSIS OF THE RESULTS}

Table 1 shows the image compression result in this RMSE, PNR and CR is computed for Wavelet and Curvelet transform.
The basic idea is that any face image is a unique linear combination of these independent components.

D. Non-negative matrix factorization (NMF)

Non-negative matrix factorization (NMF) is a newly advanced technique for finding parts, and it is based on representations of non-negative data .Given a non-negative data matrix X, This algorithm finds an approximate factorization $\mathrm{X}=\mathrm{W} . \mathrm{H}$ into non-negative factors of $\mathrm{W}$ and $\mathrm{H}$. This representation purely additive, in this contrast to many other linear representations algorithms such as PCA or ICA also used but PCA and ICA offer results complicated to understand .W and $\mathrm{H}$ are chosen as the matrix that minimize computation error. The importance of NMF is that it has capacity of obtaining significant features in collections of real biological data. When applied to $\mathrm{X}=$ Faces, NFM generates base vectors that are intuitive the unique features of the faces .

Table 1. Image Compression Analysis

\begin{tabular}{|l|c|c|c|c|c|c|}
\hline \multirow{2}{*}{$512 \times 512$} & \multicolumn{2}{|l}{$\begin{array}{l}\text { Wavelet Transform with Scalar } \\
\text { Quantization }\end{array}$} & \multicolumn{2}{l|}{$\begin{array}{l}\text { Curvelet Transform with } \\
\text { Quantization }\end{array}$} \\
\cline { 2 - 8 } & RMSE & PSNR & CR & RMSE & PSNR & CR \\
\hline Image 1 & 8.60 & 29.4 & 60.3 & 4.97 & 34.3 & 85.1 \\
\hline Image 2 & 8.12 & 29.9 & 60.1 & 4.91 & 34.33 & 87.2 \\
\hline Image 3 & 8.52 & 26.1 & 50.2 & 5.35 & 34.54 & 88.1 \\
\hline
\end{tabular}

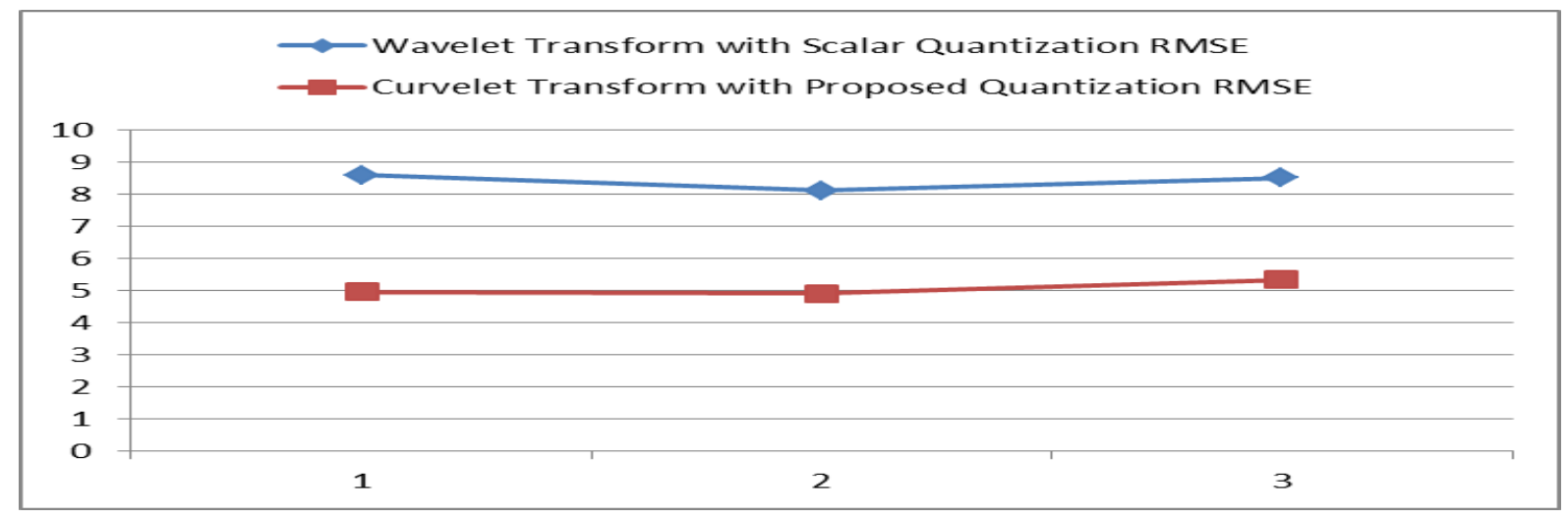

Fig 4:. RMSE/Images. 


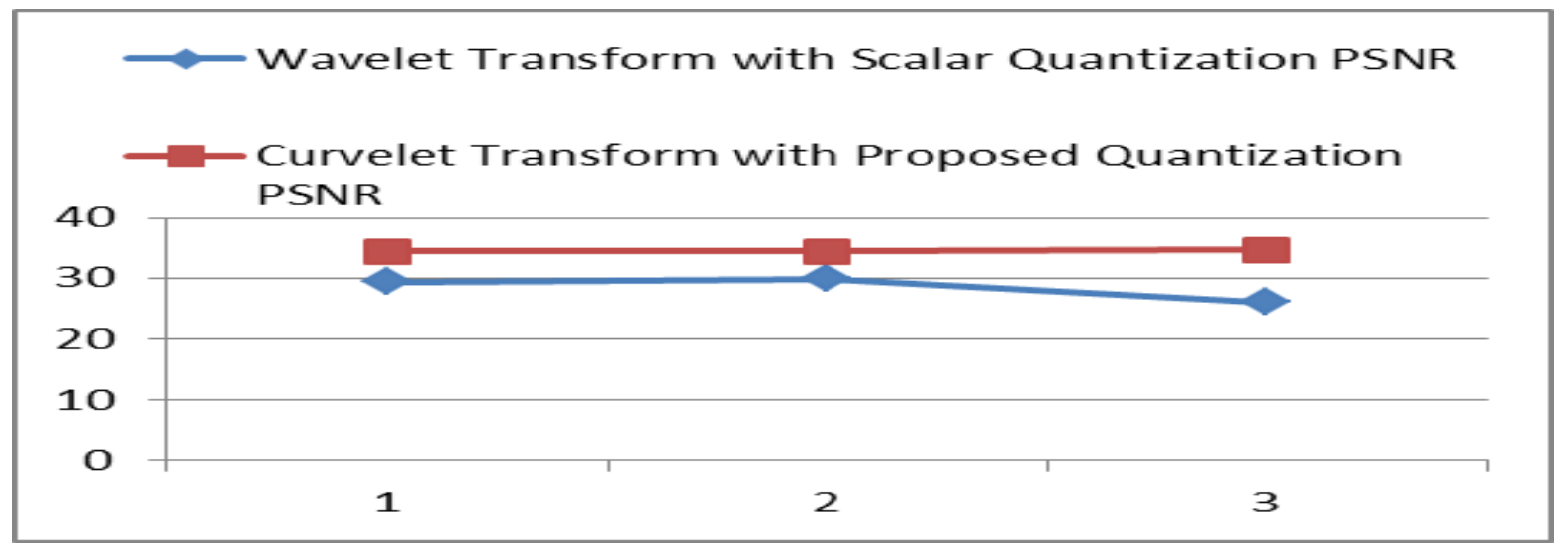

Fig 5: PSNR/Images

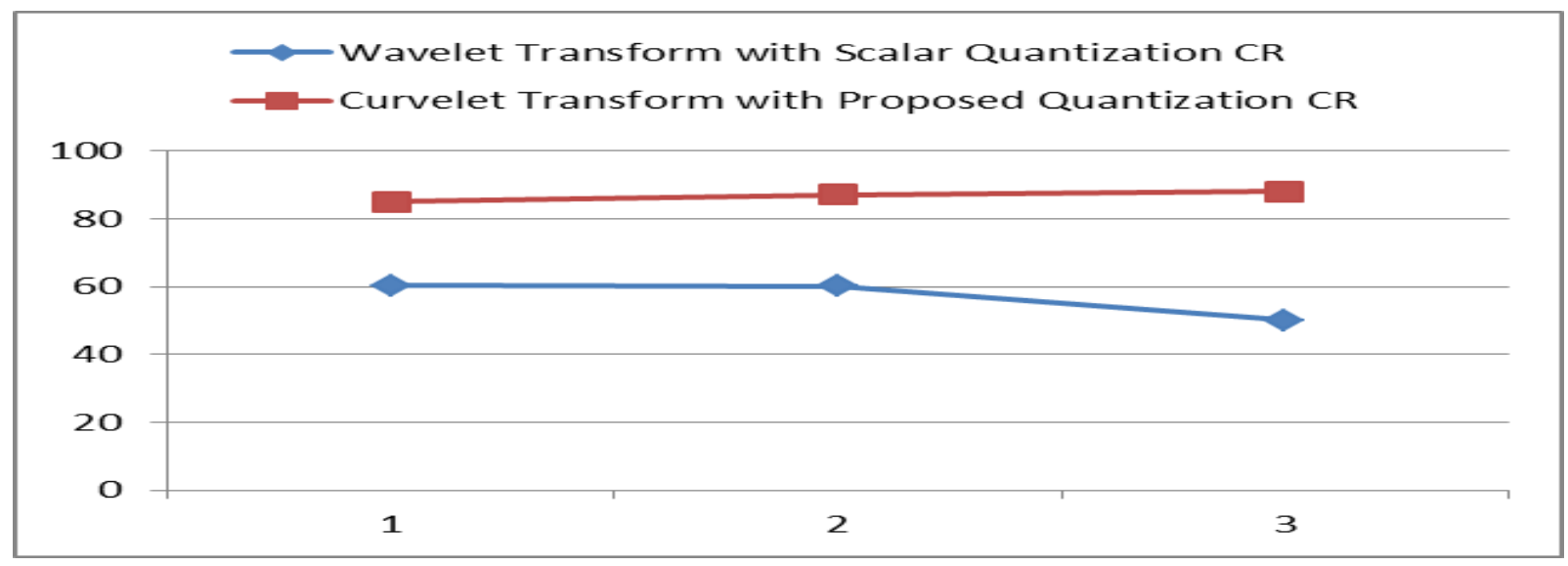

Fig 6: CR/Images.

RMSE comparison of the proposed curvelet transform and the wavelet transform is shown in Table 1. It is observed that the proposed curvelet transform with proposed quantization is observed to have lesser RMSE when compared with the wavelet based scalar quantization image compression approach.

PSNR values of the proposed curvelet transformation with proposed quantization and the compression technique based on wavelet transformation and scalar quantization. It is observed that the PSNR values of the proposed compression technique which uses curvelet transform are observed to be higher than the wavelet transform based image compression. The compression ratio $\mathrm{CR}$ of the proposed technique is observed to be very significant when compared with the existing approach. The standard images of size $512 \times 512$ are taken. For all the images the value of proposed Curvelet
Transform with proposed Quantization approach attains a compression ratio is higher than the wavelet transform based image compression.

The works on face recognition accuracy using curvelet transform that exist in prose are not yet complete and do not fully understand the capability of curvelet transform for face recognition.To compare the curvelet transform with PCA, LDA ICA and NMF. In given Table 3 shows the results are obtained from ORL database. It is clear that the comparison gives the recognition rate for all algorithms based on changed images the recognition graph is shown in figure $7 \& 8$ PCA is $96.6 \%$, LDA is $98.1 \%$ and recognition rate for ICA is $97.5 \%$ for the corresponding 200 images.; hence, there is much scope of improvement in terms of both recognition accuracy and curvelet-based methodology.

Table 2. Recognition rate of PCA, LDA, ICA

\begin{tabular}{|c|c|c|c|}
\hline No.of Images & PCA & LDA & ICA \\
\hline 5 & 91.43 & 91.98 & 90.1 \\
\hline 7 & 93.88 & 94.2 & 90.4 \\
\hline 8 & 94.0 & 95 & 90.9 \\
\hline 10 & 94.86 & 95.8 & 92.3 \\
\hline
\end{tabular}


Table 3. Recognition rate of PCA, LDA ,ICA, NMF with Curvelet Transform

\begin{tabular}{|c|c|c|c|c|}
\hline No of Images & PCA+ Curvelet & $\begin{array}{c}\text { LDA+ } \\
\text { Curvelet }\end{array}$ & $\begin{array}{c}\text { ICA+ } \\
\text { Curvelet }\end{array}$ & $\begin{array}{c}\text { NMF + } \\
\text { Curvelet }\end{array}$ \\
\hline 40 & 92.6 & 93.3 & 90.1 & 90 \\
\hline 60 & 94.2 & 95.2 & 91.5 & 91.5 \\
\hline 120 & 95.6 & 96.2 & 92.4 & 92.7 \\
\hline 160 & 96.4 & 97.5 & 94.3 & 94.7 \\
\hline
\end{tabular}

Graph Between Recognition Rate /No of Images For Table $2 \& 3$ is shown in figure:7\&8.

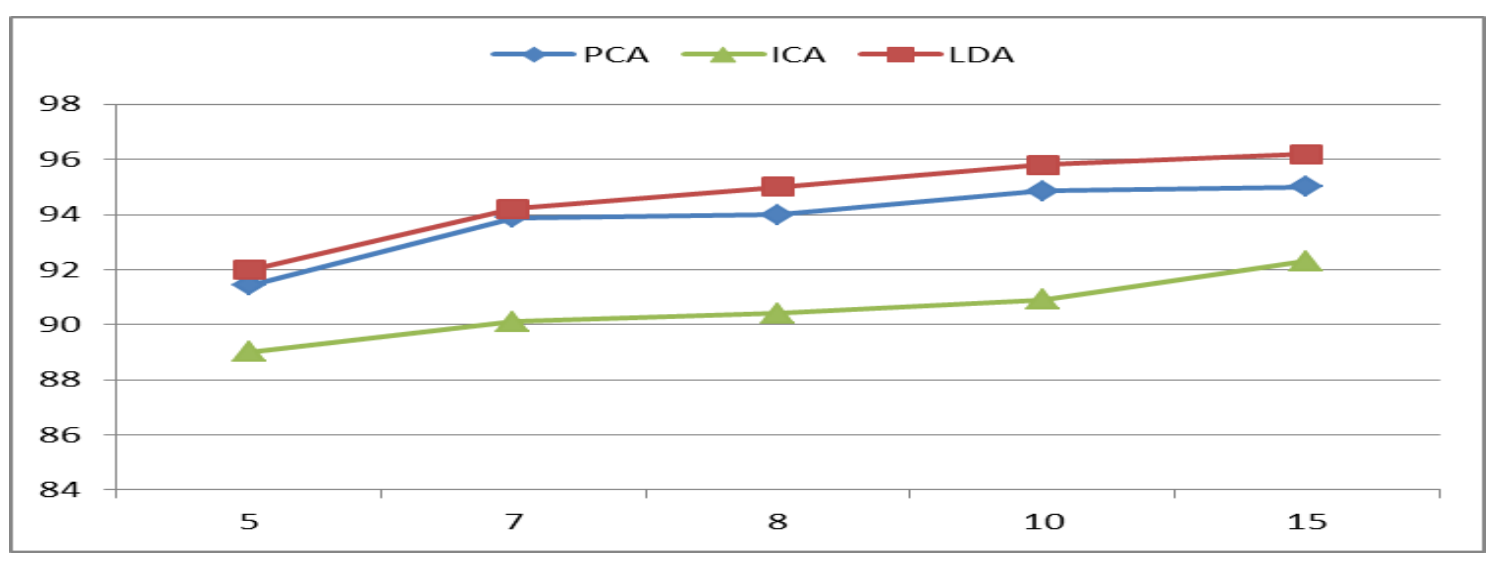

Fig 7:. Recognition Rate /No. of Images.

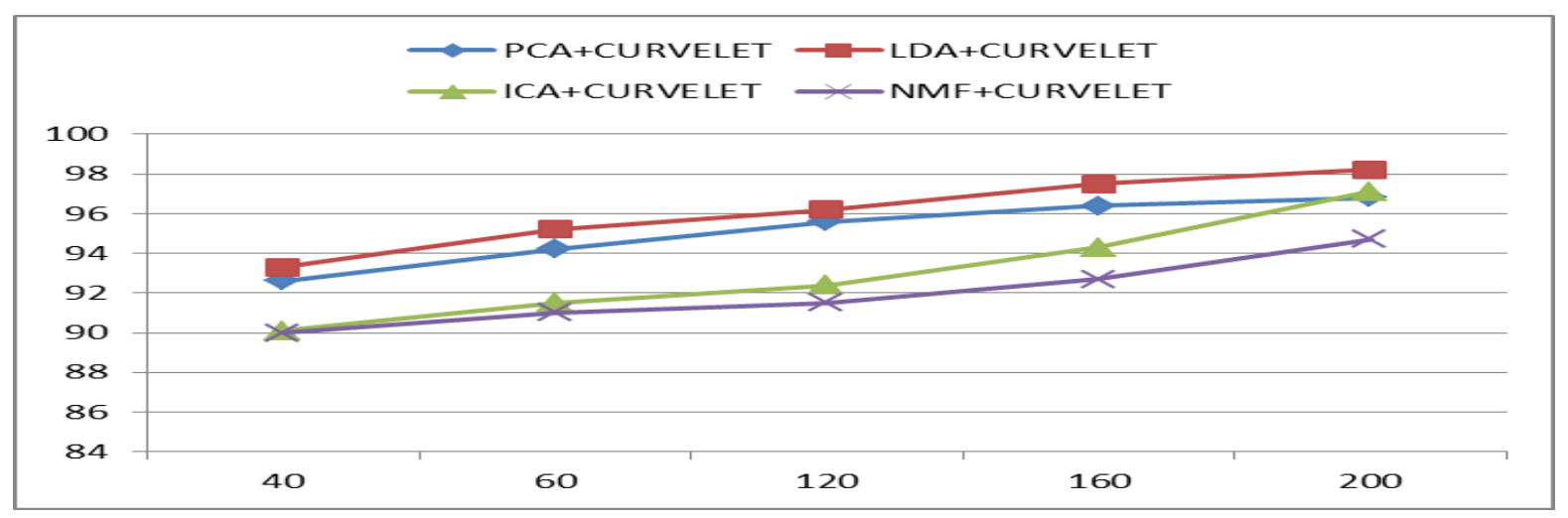

Fig 8:. Recognition Rate /No. of Images.

\section{CONCLUSION}

In this paper we have analyse two algorithm. In first algorithm the Image Compression using Curvelet Transform with proposed quantizer based on dead zone, In second algorithm three subspace projection which are PCA, LDA, and ICA are studied. PCA, LDA, ICA and NMF are combined with curvelet transform. Our analysis is based on the ORL face dataset, recognition performance for all the algorithms is 
evaluated. The experimental results gives the detail that LDA and LDA+Curvelet. These are dimensionality reduction technique, and can be employed for face recognition very well. Curvelets can be applied to diverse fields so that revolutionary results can be obtained. This shows that the Curvelet Transforms are more suitable for the image data to represent the singularities over geometric structures in the image, Than the wavelet counterpart. Curvelet is designed to age data to represent the singularities on curves. Wavelets are effective for point singularities.

\section{FUTURE SCOPE}

1) The computational cost of curvelets is higher than that of wavelets. Software that would be able to implement fast curvelet algorithm that reduce the computational time have continued to be a growing area of research.

2) Rotation and scale invariance will be investigated to further improve curvelet retrieval performance. Application of curvelet feature in color image retrieval and semantic learning will also be investigated.

3) Curvelet tansform can we use for Criminal investigation and forensic application in this the image recognition from all angle of body and motion of body also become $\mathrm{s}$ area of research.

\section{REFERENCES}

[1] E. Gomathi and K. Baskaran "Face Recognition Fusion Algorithm Based on Wavelet" European Journal of Scientific Research ISSN 1450-216X Vol.74 No.3, pp. 450-455,2012

[2] E. Candès and L. Demanet, "The curvelet representation of wave propagators is optimally sparse," Commun. Pure Appl.Math. pp. 1472-1528, vol. 58, no. 11,2005

[3] Nilima D. Maske, Wani V. Patil "Comparison of Image Compression using Wavelet for Curvelet Transform \& Transmission over Wireless Channel" International Journal of Scientific and Research Publications, , Issue 5, ISSN 2250-3153 Volume 2, May 2012.

[4] C. Villegas-Quezada and J. Climent, "Holistic face recognition using multivariate approximation, genetic algorithms and adaboost classifier: preliminary results," World Academy of Science, Engineeringand Technology, vol. 44, pp. 802-806, 2008.

[5] L. L. Shen and L. Bai, "Gabor feature based face recognition usingkernal methods," in Proceedings of the 6th IEEE International Conference on Automatic Face and Gesture Recognition (FGR '04), vol. 6, pp. 386-389, May 2004

[6] M. Zhou and H. Wei, "Face verification using gabor wavelets and AdaBoost," in Proceedings of the 18th International Conference on Pattern Recognition (ICPR '06), vol. 1, pp. 404-407, August 2006.
[7] S. Chen, X. Tan, Z. H. Zhou, and F. Zhang, "Face recognition from a single image per person: a survey," Pattern Recognition, vol. 39, no. 9, pp. 1725-1745, 2006.

[8] Y. Gao and M. K. H. Leung, "Face recognition using line edge map," IEEE Transactions on Pattern Analysis and Machine Intelligence, vol. 24, no. 6, pp. 764-779, 2002.

[9] C. BenAbdelkader and P. Griffin, "A local region-based approach togender classification from face images," in Proceedingsof the IEEE Computer Society Conference on ComputerVision and Pattern Recognition, vol. 3, pp. 52$57,2005$.

[10] M. Turk, A. Pentland, Face Recognition using Eigenfaces, Proc. Computer Vision and Pattern Recognition, pp 586591,1991

[11] T. Ahonen, A. Hadid, and M. Pietikainen, "Face description withlocal binary patterns: application to face recognition," The IEEE Transactions on Pattern Analysis andMachine Intelligence,vol. 28, pp. 2037-2041, 2006.

[12] G. C. Feng, P. C. Yuen, D. Q. Dai, Human Face Recognition using PCA on Wavelet Subband, Journal of Electronic Imaging, Vol. 9, Issue 2, pp 226-233, 2000

[13] R. Gottumukkal and V. K. Asari, "An improved face recognition technique based on modular PCA approach," Pattern Recognition Letters, vol. 25, no. 4, pp. 429-436, 2004.

[14] C. C. Liu and D. Q. Dai, "Face recognition using dualtree complex wavelet features," IEEE Transactions on Image Processing, vol. 18, no. 11, pp. 2593-2599, 2009.

[15] Truong T. Nguyen and Hervé Chauris "Uniform Discrete Curvelet Transform" IEEE TRANSACTIONS ON SIGNAL PROCESSING,3618-3634, VOL. 58, NO. 7, JULY 2010.

[16] P S Arun Kumar, "Implementation of Image Compression Algorithm using Verilog with Area, Power and Timing Constraints", 2009.

[17] Balasubramanian, R.; Bouman, C.A.; Allebach, J.P.; "Sequential scalar quantization of vectors:an analysis", IEEE Transactions on Image Processing, Volume: 4, Issue: 9, Page(s): 1282 -1295, 1995.

[18] Ching-Min Cheng; Soo-Chang Pei; "Dependent scalar quantization of color images", IEEE Transactions on Circuits and Systems for Video Technology, Volume: 5 , Issue: 2, Page(s): 124 - 139, 1995.

[19] C;Marmotton F, Ziad,A.O;Oliver,"Wavelet image coding with adaptive dead-zone selection:application to JPEG2000",Proceedings of International Conferenc on Image processing,Volome:3,2002. 